\title{
THE GREEN TEA POLYPHENOL EPIGALLOCATECHIN-3-GALLATE (EGCG) ATTENUATES SKELETAL MUSCLE ATROPHY IN A RAT MODEL OF SARCOPENIA
}

\author{
B.M. MEADOR ${ }^{1}$, K.A. MIRZA², M. TIAN¹, M.B. SKELDING ${ }^{1}$, L.A. REAVES ${ }^{1}$, N.K. EDENS ${ }^{1}$, \\ M.J. TISDALE ${ }^{2}$, S.L. PEREIRA ${ }^{1}$ \\ 1. Abbott Nutrition, Research and Development, Columbus, OH, USA; 2. Nutritional Biomedicine, School of Life and Health Sciences, Aston University, Birmingham, UK. \\ Corresponding author: Suzette L. Pereira, Ph.D. Abbott Nutrition, Strategic R\&D, RP4-3, 3300 Stelzer Road, Columbus, OH 43219, suzette.pereira@abbott.com, Tel: 1-614-624-4625, \\ Fax: 1-614-727-4625
}

\begin{abstract}
Objective: Sarcopenia - the loss of muscle mass and functionality occurring with age - is a pervasive problem with few effective treatments beyond exercise. We examined the ability of the green tea catechin, epigallocatechin-3-gallate (EGCg), to impact muscle mass and the molecular pathway involved in muscle atrophy in a rat model of sarcopenia. Methods: 20-month-old Sprague-Dawley rats were treated for 8 weeks with control diet or control plus $200 \mathrm{mg} / \mathrm{kg}$ body weight of EGCg diet. Results: EGCg-supplemented animals had significantly greater gastrocnemius muscle mass than the aged controls, and showed a trend for increased muscle fiber cross-sectional areas (CSA) $(\mathrm{p}=0.06)$. These changes were associated with significantly lower protein expressions of the intramuscular 19S and 20S proteasome subunits and the MuRF1 and MAFbx ubiquitin ligases in the EGCg-treated animals. Proteasome activity as determined by 'Chymotrypsin-like' enzyme activity was also significantly reduced by EGCg. Muscle mRNA expression of IL-15 and IGF-1 were significantly increased in the EGCg group vs. the aged controls. In comparison to younger adult animals (6 month), the protein expression of 19S, 20S, MuRF1, MAFbx, and myostatin were increased between approximately 4- and 12-fold in the aged controls, but only up to $\sim 2$-fold in the aged EGCg animals. Conclusions: EGCg supplementation was able to preserve muscle in sarcopenic rats, partly through attenuating protein degradation via the ubiquitinproteasome pathway, together with increased expression of anabolic factors.
\end{abstract}

Key words: Chymotrypsin-like activity, IGF-1, myostatin, ubiquitin proteasome pathway.

\section{Introduction}

Sarcopenia - the loss of lean body mass and function with aging - is a primary concern for the world's increasingly aged population. After the age of 40 , the loss of lean body mass occurs at approximately $8 \%$ per decade, with further acceleration at older ages $(5,21)$. This decline is linked to decreased quality of life through numerous mechanisms, including reduced strength and energy, as well as an increased risk for frailty and the further complications - such as falls and fractures-associated with the frailty syndrome (21).

Various interventions have been investigated for their ability to slow functional decline with aging, of which exercise seems to be the most effective to date (9). However, exercise may not always be a feasible option, especially in situations involving physical impairments. Thus other interventions need to be considered that can address and prevent muscle wasting in the sarcopenic population. Since the cause of sarcopenia is multifactorial, such an intervention may have to address inflammation, oxidative stress, and other factors.

Epigallocatechin gallate (EGCg) is the most abundant catechin in green tea, accounting for approximately $60 \%$ of catechin content, and has strong antioxidant and antiinflammatory properties (26). The biological effects of green tea and its extracts are diverse, and there are indications that it has an impact on lean body mass. For instance, it has been shown to improve muscle function in a mouse model of muscular dystrophy $(7,12)$ and to attenuate atrophy in cancer cachexia (34). Additionally, there is one clinical trial demonstrating that green tea intake can improve muscle strength - as measured by wall sit times - in older humans (33). These prior data suggest a role for green tea catechins in the treatment and prevention of muscle-associated disorders.

Mechanisms through which green tea catechins may affect outcomes in skeletal muscle have not been well characterized. However, research in cancer models has demonstrated that catechins can suppress proteasome activity $(28,23)$, which also presents an appropriate target for investigation in sarcopenia. The ubiquitin proteasome proteolytic (UPP) pathway plays a major role in protein degradation in skeletal muscle, and has been shown to have increased expression in aging muscle (16) and is believed to contribute to sarcopenia (2).

The Sprague-Dawley rat is a well-established animal model of sarcopenia, demonstrating decrements in muscle mass, fiber CSA and neuromuscular function with aging, starting at age of 20 months $(2,13)$. In particular, type II muscle fibers tend to undergo atrophy in aged skeletal muscle, and it accounts for $92 \%$ of gastrocnemius muscle $(36,37)$. Therefore, this investigation aimed to examine the impact of 8-weeks' EGCg supplementation on gastrocnemius muscle in aged 


\section{EGCG SUPPLEMENTATION IN SARCOPENIA}

Sprague Dawley rats. Gastrocnemius muscle was collected for assessment of muscle mass and fiber CSA, as well as the expression of various proteins and genes associated with protein degradation and muscle anabolism. In addition, we compared results obtained from this study to a young animal cohort, to understand aging-related changes in protein and gene expression in muscle.

\section{Methods}

\section{Model}

Twenty-four aged (20 month) male Sprague Dawley rats (Harlan Laboratories) were randomly assigned to one of two treatment diet groups after blocking for initial bodyweight. Rats were housed individually for the 8-week duration of the study. Blood withdrawals were performed at the start of the study to provide baselines. Bodyweights were recorded weekly, including initial and final weights. At the end of the study, rats were sacrificed by $\mathrm{CO} 2$ asphyxiation after fasting for 16 hours, blood was drawn by heart puncture and gastrocnemius muscles were removed immediately and frozen for further analyses.

In addition to the aged rats, a small set $(n=3)$ of younger adult rats (6 months) was used for western blot analyses of UPP components (19S, 20S, MuRF1, MAFbx) and myostatin to provide a baseline for examining the changes seen with aging, and the extent - if any - to which EGCg treatment was able to reverse these changes. These animals were fed standard chow diets and housed in the study facilities for one week prior to sacrifice.

All animal procedures were approved by the Ohio State University animal care committee (IACUC protocol 08A0040). Animals were housed in Ohio State's AAALAC-accredited, OLAW-approved animal-care program facilities, with oversight and care by the program's attending veterinarian.

\section{Diet}

Diets were prepared by Research Diets Inc. (New Brunswick, NJ). The American Institute of Nutrition rodent diet AIN-93M was used as the control diet (12.4\% protein; $68.4 \%$ carbohydrate; $4.1 \%$ fat by weight). Treatment diet was AIN-93M with the addition of 5mg EGCg (Teavigo, DSM) per gram of diet, to achieve an approximate daily dosing of $200 \mathrm{mg}$ $\mathrm{EGCg} / \mathrm{kg}$ body weight (assuming average food consumption of $20 \mathrm{~g} /$ day/rat). Rats were fed their assigned diet ad libitum for 8 weeks. Rats were checked daily to ensure adequate food availability, and intake was assessed weekly. Diets were handled under low light conditions and stored at $4{ }^{\circ} \mathrm{C}$ in sealed containers.

\section{Note}

The study design also included a treatment group receiving $50 \mathrm{mg} / \mathrm{kg} /$ day EGCg. However, results are not shown for this treatment, as the results at this dosage were not as robust as the higher $200 \mathrm{mg}$ dosing and could not be duplicated in two subsequent experiments using the same model.

\section{Muscle Analyses}

At the end of the treatment period, gastrocnemius (gastroc) muscles were isolated from the hindlimbs of each rat. Muscles were frozen in liquid-nitrogen-cooled isopentane for histological analyses or snap-frozen in liquid nitrogen for protein and gene analysis.

Histology-prepared gastroc samples were analyzed by Seventh Wave Laboratories (Chesterfield, MO) for fiber crosssectional area. Control and EGCg groups consisted of 11 and 12 rats, respectively. Eight micron sections from muscle samples were cut, mounted, stained using hematoxylin \& eosin (H\&E), and analyzed. Approximately 400 fibers were measured per sample.

Partial snap-frozen gastrocs were analyzed by Syngene (India) for the gene expression of IGF-1, IL-15, and IL-15R. Briefly, total RNA was extracted and cDNA synthesized. Q-PCR analysis was performed using TaqMan gene expression assays which were pre-designed and custom synthesized (Applied Biosystems, USA). The average of the 4 endogenous controls B2M (beta 2 microglobin), Hprt1 (hypoxanthine phosphoribosyltransferase 1), GAPDH (Glyceraldehyde 3-phosphate dehydrogenase) and ACTB (Actin B) was used as the house-keeping baseline for fold change calculations. Gene expression analysis was performed in duplicate and examined by the delta-delta-CT method.

For protein analyses, total soluble-fraction protein was extracted from the gastroc and assessed for protein content by western blotting. Muscle samples were homogenized in ice cold QS1 buffer (10mM Trizma Base- $\mathrm{HCl}$ pH $8.0,0.5 \mathrm{mM}$ PMSF, $1 \mathrm{mM}$ DTT, $0.5 \mathrm{mM}$ EGTA) at a ratio of $1 \mathrm{~g}$ tissue per $5 \mathrm{~mL}$ buffer, and then centrifuged at $4000 \mathrm{~g}$ for 20 minutes. The formed pellet was removed, and the supernatant centrifuged again at $4500 \mathrm{rpm}$ for $20 \mathrm{~min}$. The resulting supernatant was used for protein concentration measurement by using standard Bradford protein assay protocols (Bio-Rad). 10 $\mu \mathrm{g}$ of protein per sample were loaded and run on $10 \%$ SDS polyacrylamide gels. Proteins were electrotransferred to presoaked nitrocellulose membranes. Gel-transfer apparatus was kept on ice during the 2-hour transfer protocol. After transfer the membranes were blocked in $50 \mathrm{~mL}$ blocking buffer $(5 \%$ Marvel) for 2 hours. Membranes were subsequently rinsed with $50 \mathrm{~mL} 0.5 \%$ PBS-tween, then incubated with the primary antibody diluted in $0.1 \%$ PBS-Tween, overnight at $4{ }^{\circ} \mathrm{C}$. Primary antibodies: 19S mouse monoclonal, 1:1000 (Affiniti Research Products, Exeter, UK), 20S mouse monoclonal, 1:1000, (Affiniti Research Products), MuRF1 rabbit polyclonal antisera, 1:200 (Santa Cruz Biotechnology, Inc., Santa Cruz, CA), MAFbx rabbit polyclonal antisera, 1:200 (Santa Cruz Biotechnology, Inc.), myostatin rabbit polyclonal, 1:50 (Abcam, Cambridge, UK). After incubation, the membranes were rinsed with $0.1 \%$ PBS-Tween, then incubated for 1 hour in secondary antibody (1:1000 dilutions in 0.1\% PBS-Tween). 


\section{THE JOURNAL OF FRAILTY \& AGING}

Membranes were then cycled through three 10-minute washes in $0.1 \%$ PBS-Tween. Bands were visualized on the X-ray film using developer and fixer. Band quantification was performed using ImageJ software, version 1.46 (National Institute of Health, USA), with values normalized by tubulin loading.

Proteasome activity was measured by 'Chymotrypsin-like' enzyme activity assay. Briefly, $10 \mu \mathrm{l}$ of the supernatant was added in triplicate to a black, flat-bottomed polystyrene plate (Corning, Inc. New York), followed by $10 \mu$ of lactacystin added to half the plate. $100 \mu \mathrm{l}$ of the chymotrypsin substrate (N-Succinyl-Leu-Leu-Val-Tyr-7-Amido-4-Methyl-Coumarin) was then added to every well. The plate was then read on a fluorometer set for reading $360 \mathrm{~nm}$ excitation and 460nm emission. The plate was then incubated at room temperature for $1 \mathrm{~h}$ with gentle shaking. After an hour the plate was read again at an excitation wavelength of $360 \mathrm{~nm}$ and an emission wavelength of $460 \mathrm{~nm}$. The chymotrypsin activity was measured as activity per $\mu \mathrm{g}$ of protein. Study personnel were blinded to treatment groups for any of the analyses.

\section{Blood Analyses}

Blood withdrawals were performed by tail bleeds at baseline, and by cardiac puncture at the end. Glucose was assessed immediately with a Precision PCx Point of Care glucometer (Abbott Laboratories). Whole blood was immediately centrifuged and the serum extracted. Insulin levels were assessed by ELISA (ALPCO, Salem).

\section{Statistical Analyses}

Differences in mean values between control and EGCg groups were determined by student's t-test. For comparisons including the younger animal group, one-way ANOVAs, followed by Tukey-Kramer multiple-comparison tests were performed. p-values $<0.05$ were considered significant. Results were expressed as mean values \pm SEM for at least three replicate experiments.

\section{Results}

\section{Food Intake, Glucose \& Insulin}

Total diet consumption over the course of the study was not significantly different between groups, at $1166 \mathrm{~g} \& 1185 \mathrm{~g}$ for the control and EGCg groups, respectively. Additionally, there were no significant differences when individual weekly collection periods were compared between groups (data not shown).

Fasted glucose and insulin did not differ between groups at baseline or at the end of the study. While both groups showed increased fasted insulin levels at end of the study, this change was not significantly different between groups and appears to have been an effect of aging, rather than treatment (Table 1).

\section{Body Weight, Muscle Weight and Histology}

There was no significant difference in body weight between groups at baseline. Both aged groups significantly increased their bodyweights over the course of the study, although the increases were not significantly different between groups (Table 1).

EGCg supplementation resulted in significant increase in gastrocnemius muscle wet weight by $4.9 \%$ compared to aged control animals (Table 1). The gastroc masses were not significantly different between groups when expressed as a ratio to total bodyweight.

A trend for increased muscle fiber cross-sectional area was also found for EGCg supplementation, with $8.4 \%$ greater CSA in EGCg group than control group ( $\mathrm{p}=0.06)$ (Table 1).

Table 1

Effect of EGCg treatment on body weight, muscle weight, blood glucose and insulin in aged rats

\begin{tabular}{|c|c|c|c|c|}
\hline & Control $(n=12)$ & $\operatorname{EGCg}(n=12)$ & p-value* & p-value $\dagger$ \\
\hline Initial body weight (g) & $592 \pm 6.7$ & $591 \pm 9.0$ & 0.93 & \\
\hline Final body weight (g) & $620 \pm 12.6$ & $640 \pm 13.9$ & 0.31 & \\
\hline Change (Body weight, g) & $28.3 \pm 11$ & $48.8 \pm 7.6$ & 0.14 & $0.06(\mathrm{C}) ; 0.007(\mathrm{E})$ \\
\hline Final Gastroc wt (g) & $2.85 \pm 0.06$ & $2.99 \pm 0.03$ & 0.04 & \\
\hline Gastroc/BW (mg/g) & $4.6 \pm 0.1$ & $4.7 \pm 0.1$ & 0.53 & \\
\hline Fiber CSA $\left(\mu \mathrm{m}^{2}\right) \neq$ & $3087 \pm 274$ & $3347 \pm 349$ & 0.06 & \\
\hline Initial Glucose (mg/dL) & $86.5 \pm 1.7$ & $87.3 \pm 1.9$ & 0.76 & \\
\hline Final Glucose (mg/dL) & $83.7 \pm 3.6$ & $88.5 \pm 2.0$ & 0.25 & \\
\hline Change (Glucose, mg/dL) & $-2.8 \pm 3.3$ & $1.2 \pm 1.4$ & 0.26 & $0.49(\mathrm{C}) ; 0.67(\mathrm{E})$ \\
\hline Initial Insulin (pM) & $196 \pm 24$ & $153 \pm 11$ & 0.11 & \\
\hline Final Insulin (pM) & $229 \pm 37$ & $174 \pm 40$ & 0.19 & \\
\hline Change (Insulin, pM) & $33 \pm 24$ & $21 \pm 14$ & 0.66 & $0.46(\mathrm{C}) ; 0.33(\mathrm{E})$ \\
\hline
\end{tabular}

Values are presented as mean \pm SEM.; $*$ EGCg vs. control; $\uparrow$ Within group change from baseline: $\mathrm{C}=\mathrm{Control} ; \mathrm{E}=\mathrm{EGCg} ; \ddagger \mathrm{Control} \mathrm{n}=11, \mathrm{EGCg} \mathrm{n}=12$ 


\section{Ubiquitin Proteasome Pathway}

The protein expressions of the proteasome 20S core particle, $19 \mathrm{~S}$ regulatory subunit, and the ubiquitin ligases MuRF1-1 and MAFbx were all found to be significantly lower in the EGCgtreated animals than the aged controls. Average treatment values of 20S, 19S, MuRF1, and MAFbx were 19\%, 16\%, $17 \%$, and $14 \%$, respectively, of aged control values (Figure 1). Additionally, the 'chymotrypsin-like' enzyme activity was significantly lower in the EGCg group, averaging $14 \%$ of aged control values (Figure 2). When normalized to $20 \mathrm{~S}$ protein levels, the activity was not significantly different from control, implying that the decrease in 'chymotrypsin-like' activity was likely due to reductions in number of proteasomes.

\section{Figure 1}

Ubiquitin Proteasome Pathway (UPP) components and regulator protein expression as measured by western blotting in aged control vs. aged EGCg rats $(\mathrm{n}=12$ each)

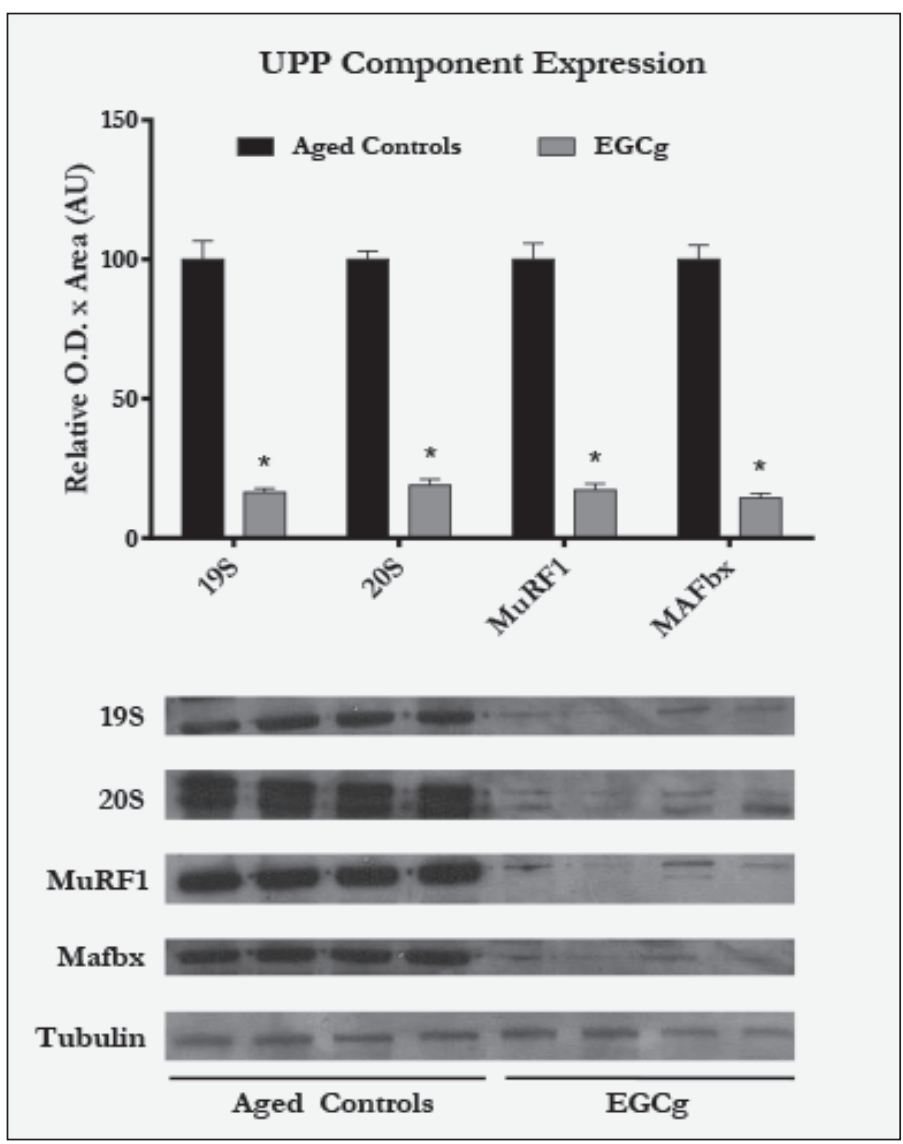

Bar graph presents mean values $\pm \mathrm{SEM},{ }^{*} \mathrm{p}<0.05$ vs. corresponding aged control rats Representative blots from four animals per group are shown; tubulin was used as an endogenous control.

\section{Anabolic \& Catabolic Factors}

Intramuscular IGF-1 mRNA expression was significantly higher (37\%) in EGCg-treated animals than controls. IL-15 mRNA was also significantly increased (26\%) by EGCg, while the increase in IL-15R (25\%) was not statistically significant (Figure 3).
Figure 2

Chymotrypsin-like enzyme activity in aged control vs. EGCg rats. "Absolute" represents activity value normalized to total lysate protein content, "Normalized" represents activity value normalized to $20 \mathrm{~S}$ protein content and $(\mathrm{n}=4$ for aged controls, $\mathrm{n}=11$ for $\mathrm{EGCg}$ )

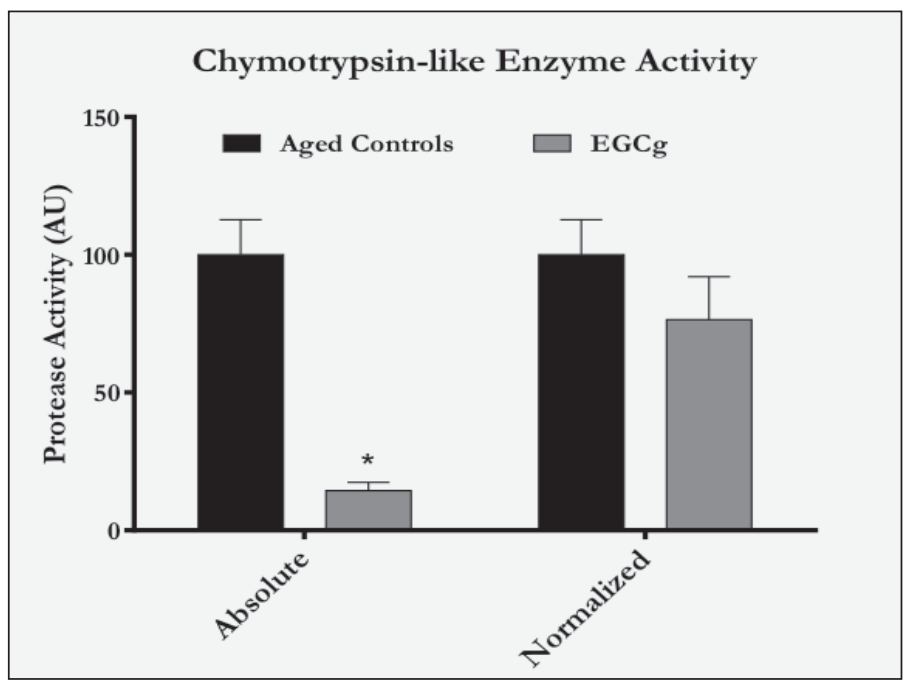

* Bar graph presents mean values $\pm \mathrm{SEM}, \mathrm{p}<0.05$ vs. corresponding aged control.

Figure 3

Anabolic gene expression as measured in muscle lysates by qPCR and ( $\mathrm{n}=11$ for aged controls, $\mathrm{n}=8$ for $\mathrm{EGCg}$ )

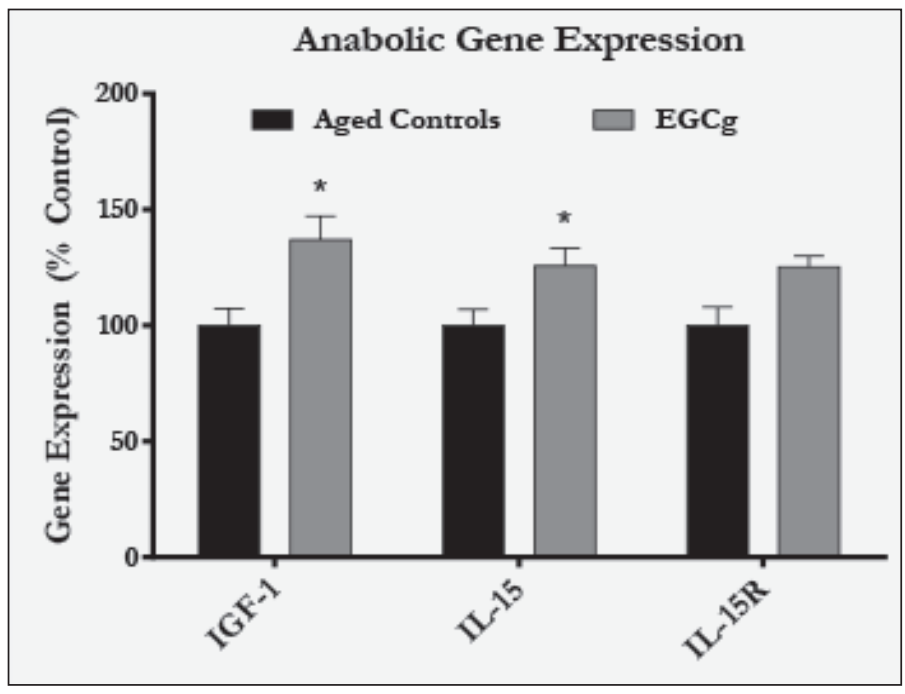

* Bar graph presents mean values \pm SEM, $\mathrm{p}<0.05$ vs. corresponding aged control.

\section{Comparison with Young Rats}

Compared to young animals, the protein expression of the UPP components (20S, 19S, MuRF1, and MAFbx) in muscle was increased by 4-12 fold in aged controls, and EGCg treatment was able to significantly attenuate these increases (Table 2). 


\section{THE JOURNAL OF FRAILTY \& AGING}

Table 2

Protein levels of UPP components and myostatin

\begin{tabular}{lccc}
\hline & $\begin{array}{c}\text { Young } \\
(\mathbf{n = 3})\end{array}$ & $\begin{array}{c}\text { Aged } \\
(\mathbf{n = 3})\end{array}$ & $\begin{array}{c}\text { Aged + EGCg } \\
(\mathbf{n = 3})\end{array}$ \\
\hline $20 \mathrm{~S}$ & $100 \pm 33$ & $443 \pm 6^{*}$ & $213 \pm 78 \dagger$ \\
19S & $100 \pm 19$ & $1201 \pm 67^{*}$ & $230 \pm 38^{*} \dagger$ \\
MuRF1 & $100 \pm 11$ & $967 \pm 32^{*}$ & $207 \pm 17 * \dagger$ \\
MAFbx & $100 \pm 8$ & $1084 \pm 8^{*}$ & $219 \pm 51 \dagger$ \\
Myostatin & $100 \pm 14$ & $663 \pm 30^{*}$ & $102 \pm 22 \dagger$ \\
\hline All data is relative optical density $x$ area in arbitrary units, and presented as mean \pm SEM; \\
$*$ p $<0.05$ vs. corresponding young group; $\dagger$ p $<0.05$ vs. Aged group.
\end{tabular}

Similarly, intramuscular protein expression of myostatin was significantly elevated in the aged control animals compared to the young animals (6-fold). And this elevated expression with aging was prevented by EGCg treatment (Table 2).

\section{Discussion}

In the current study using the aged Sprague-Dawley rat model of sarcopenia, we found that EGCg supplementation at $200 \mathrm{mg} / \mathrm{kg}$ body weight for 8 weeks resulted in greater gastrocnemius muscle mass, with trended increase in muscle fiber cross-sectional areas. These changes were not associated with changes in food intake. In addition, EGCg supplementation resulted in significant reduction in the intramuscular protein level of proteasome subunits (19S, 20S) and ubiquitin ligases (MAFbx, MuRF1), as well as the chymotrypsin activity of proteasome. Intramuscular gene expression of the anabolic factors IGF-1 and IL-15 was found to be greater in EGCg treated rats.

Previous work has shown benefits of EGCg on fat loss (19), while more recent studies have investigated its effect on muscle. Green tea extracts and EGCg were shown to increase muscle performance in a mouse model of muscular dystrophy $(7,12)$, attenuate atrophy in cancer cachexia mouse model (34), and improve muscle strength in older adults (33). Our current study demonstrates EGCg benefit on aging muscle in an animal model of sarcopenia, partly through the mechanism of inhibiting the UPP pathway.

The UPP is the key proteolytic pathway for the degradation of muscle contractile proteins under catabolic conditions, e.g. aging and cancer cachexia $(2,34)$. MuRF1 and MAFbx are the critical uniquitin ligase, which are upstream of the proteasome, composed of $19 \mathrm{~S}$ and $20 \mathrm{~S}$ subunits (24). Induction of the ubiquitin ligases MuRF1 and MAFbx has been implicated as a mechanism in various atrophy states, including cancer cachexia (34), while there is controversy over the change in direction for MuRF1 and MAFbx in aging. Some work has found their expression to decrease with age $(11,14)$, while there is also evidence for enhanced expression of MuRF1 and MAFbx (2). The discrepancy may be attributed to fiber-type, species, and gender (17). Our study demonstrated that aged rats exhibited 4-12 fold increase in protein level of the UPP components (19S, 20S, MuRF1, MAFbx) compared to young adult rats (Table 2) in the gastroc muscle, which consists of Type II fiber primarily. Interestingly, these elevated protein levels were all significantly attenuated by EGCg treatment. Furthermore, 'chymotrypsin-like' enzyme activity was reduced in the EGCg-treated animals by approximately $85 \%$ compared to controls. These data together suggest that EGCg attenuates muscle protein degradation through down regulation of the key regulatory components of the UPP in aged muscle.

Myostatin is a negative regulator of muscle growth and essential for regulation of muscle mass. Known as a modulator upstream of MuRF1 and MAFbx, blocking myostatin pathwaysrepresented a therapeutic avenue to cachexia treatment (35). Here our study showed more than 6-fold increase in muscle myostatin protein level in aged compared young rats, and EGCg significantly attenuated this increase. This result suggests that blocking myostatin could also be a possible mechanism of action for EGCG to prevent muscle loss in aging.

The inhibition of UPP components by EGCg observed were accompanied by increased gene expression of intramuscular IGF-1 and IL-15. IGF-1 is a powerful anabolic factor associated with skeletal muscle hypertrophy (1). It has been shown that the induction of IGF-1 was able to inhibit muscle declines in aging (4), and therefore presents a likely mechanism through which EGCg may be exerting its effects. The induction of IGF-1 also links to the catechin's effects on the proteasome, as the expression of IGF-1 has been shown to be a powerful inhibitor of MuRF1 and MAFbx activation (32). IL-15 has been found to have both anabolic and anti-catabolic effects in skeletal muscle in in vitro (31) and in vivo (8). Originally, in vitro studies using C2 myoblasts demonstrated that IL-15 had no effect on myoblast differentiation, but it stimulated the myosin heavy chain accumulation by differentiated myoblasts and myofibers (31), suggesting IL-15's anabolic role in muscle hypertrophy. Subsequently, IL-15 treatment was shown to partly inhibit skeletal muscle wasting in the rat model of cancer cachexia- the Yoshida AH-130 rat ascites hepatoma model, and the inhibited protein breakdown rate was associated with inhibition of the UPP pathway, including decreased mRNA expression of ubiquitin and C8 proteasome subunit (8). While it is not clear how IL-15 exerts effect in aging, there is evidence that muscle IL-15 expression decreased with aging, and the modulation of IL-15 may be an effective treatment for sarcopenia $(25,30)$. It is also possible that the reduction in UPP expression was partly a result of increased IL-15 expression.

In terms of dosage, the initial design of the current study included a lower EGCg dosage of $50 \mathrm{mg} / \mathrm{kg} /$ day, with the effect less robust and reproducible than the $200 \mathrm{mg} / \mathrm{kg}$ dose. Therefore, we focused on using the higher dose. The current dosage of $200 \mathrm{mg} / \mathrm{kg}$ rat would be equivalent to approximately $\sim 1.9 \mathrm{~g} /$ day in the average, $60 \mathrm{~kg}$ human, accounting for basic 


\section{EGCG SUPPLEMENTATION IN SARCOPENIA}

allometric differences between rats and humans - namely the 6x higher metabolic rate per mass in rats (15). Granted that while $1.9 \mathrm{~g}$ /day dose in humans is a therapeutic dose that's not achievable by drinking 2-3 cups of green tea/day, it is very close to the therapeutic doses tested in existing human trials $(10,27)$. A dosing study needs to be carried out in humans to determine the optimal dose requirements for preserving muscles during aging.

There are limitations in the current work that we would suggest as avenues of future investigations. The effect of EGCg on longitudinal changes in body composition (fat to muscle ratio) would be desirable, since there is a lot of evidence that EGCg may have a benefit on fat loss (19). Further research is also needed to determine the benefits of EGCg on muscle strength and function, which is clinically relevant in sarcopenic conditions. While not assessed in the current investigation, studies indicated that $\mathrm{EGCg}$ was able to downregulate NF-kB $(34,18)$ and FOXO (22), which are upstream modulators of the UPP pathway. Further research is needed to determine whether these factors medicate the EGCg's effect on the UPP pathway.

Funding and Authorship: This research was supported by the sponsor Abbott Nutrition. B. Meador, M. Tian, M. Skelding, L. Reaves, N. Edens, and S. Pereira are employees of Abbott Nutrition. All authors had full access to study data. The corresponding author and principal investigator S. Pereira has final responsibility for the decision to submit the publication. B. Meador, M. Tian, K. Mirza, N. Edens, M. Tisdale and S. Pereira were involved in data analysis and writing of the manuscript; $\mathrm{S}$. Pereira and N. Edens were involved in the conception; S. Pereira, N. Edens and M. Tisdale were involved in study design; M. Skelding, L. Reaves and K. Mirza were involved in data collection and collation. B. Meador, K. Mirza and M. Tian were responsible for data analysis and statistical analysis. All authors have reviewed and approved the manuscript.

Conflict of Interest: This research was supported by Abbott Nutrition. B. Meador, M. Tian, M. Skelding, L. Reaves, N. Edens, and S. Pereira are employees of Abbott Nutrition.

\section{References}

1. Adams GR, Haddad F. The relationships among IGF-1, DNA content, and protein accumulation during skeletal muscle hypertrophy. J Appl Physiol 1996; 81:2509-2516.

2. Altun M, Besche HC, Overkleeft HS, et al. Muscle wasting in aged, sarcopenic rats is associated with enhanced activity of the ubiquitin proteasome pathway. J Biol Chem 2010;285:39597-39608.

3. Balentine DA, Wiseman SA, Bouwens LC. The chemistry of tea flavonoids. Crit Rev Food Sci Nutr 1997;37:693-704.

4. Barton-Davis ER, Shoturma DI, Musaro A, et al. Viral mediated expression of insulin-like growth factor I blocks the aging-related loss of skeletal muscle function. Proc Natl Acad Sci U S A 1998;95:15603-15607.

5. Baumgartner RN. Body composition in healthy aging. Ann N Y Acad Sci 2000;904:437-448.

6. Borst SE. Interventions for sarcopenia and muscle weakness in older people. Age Ageing 2004;33:548-555.

7. Call JA, Voelker KA, Wolff AV, et al. Endurance capacity in maturing mdx mice is markedly enhanced by combined voluntary wheel running and green tea extract. J Appl Physiol 2008;105:923-932.
8. Carbo N, Lopez-Soriano J, Costelli P, et al. Interleukin-15 antagonizes muscle protein waste in tumour-bearing rats. Br J Cancer 2000;83:526531 .

9. Cruz-Jentoft AJ, Landi F, Schneider SM, et al. Prevalence of and interventions for sarcopenia in ageing adults: a systematic review. Report of the International Sarcopenia Initiative (EWGSOP and IWGS). Age and ageing 2014;43:748-759.

10. Ullmann U, Haller J, Decourt JP, et al. A single ascending dose study of epigallocatechin gallate in healthy volunteers. The Journal of International Medical Research 2003;31:88-101.

11. Deruisseau KC, Kavazis AN, Powers SK. Selective downregulation of ubiquitin conjugation cascade mRNA occurs in the senescent rat soleus muscle. Exp Gerontol 2005;40:526-531.

12. Dorchies OM, Wagner S, Vuadens O, et al. Green tea extract and its major polyphenol (-)-epigallocatechin gallate improve muscle function in a mouse model for Duchenne muscular dystrophy. Am J Physiol Cell Physiol 2006;290:C616-625.

13. Edstrom E, Altun M, Hagglund M, et al. Atrogin-1/MAFbx and MuRF1 are downregulated in aging-related loss of skeletal muscle. J Gerontol A Biol Sci Med Sci 2006;61:663-674.

14. Edstrom E, Ulfhake B. Sarcopenia is not due to lack of regenerative drive in senescent skeletal muscle. Aging Cell 2005;4:65-77.

15. US Department of Health and Human Services, FDA, Center for Drug Evaluation and Research. Guidance for Industry, Estimating the maximum safe starting dose in initial clinical trials for therapeutics in adult healthy volunteers. 2005. http://www.fda.gov/downloads/Drugs/Guidances/ UCM078932.pdf

16. Ferrington DA, Husom AD, Thompson LV. Altered proteasome structure, function, and oxidation in aged muscle. FASEB J 2005;19: 644-646.

17. Foletta VC, White LJ, Larsen AE, et al. The role and regulation of MAFbx/atrogin-1 and MuRF1 in skeletal muscle atrophy. Pflugers Arch 2011; 461: 325-335

18. Giakoustidis AE, Giakoustidis DE, Koliakou K, et al. Inhibition of intestinal ischemia/repurfusion induced apoptosis and necrosis via down-regulation of the NF-kB, c-Jun and caspace-3 expression by epigallocatechin-3-gallate administration. Free Radic Res 2008;42:180188.

19. Hursel R, Viechtbauer W, Westerterp-Plantenga MS. The effects of green tea on weight loss and weight maintenance: a meta-analysis. Intl J Obesity 2009;33:956-961.

20. Janssen I, Heymsfield SB, Ross R. Low relative skeletal muscle mass (sarcopenia) in older persons is associated with functional impairment and physical disability. J Am Geriatr Soc 2002;50:889-896.

21. Janssen I, Heymsfield SB, Wang ZM, et al. Skeletal muscle mass and distribution in 468 men and women aged 18-88 yr. J Appl Physiol 2000;89:81-88.

22. Kim H, Hiraishi A, Tsuchiya K, et al. (-) Epigallocatechin gallate suppresses the differentiation of 3T3-L1 preadipocytes through transcription factors FoxO1 and SREBP1c. Cytotechnology 2010;62:245255.

23. Kuhn DJ, Burns AC, Kazi A, et al. Direct inhibition of the ubiquitinproteasome pathway by ester bond-containing green tea polyphenols is associated with increased expression of sterol regulatory element-binding protein 2 and LDL receptor. Biochim Biophys Acta 2004;1682:1-10.

24. Lecker SH, Solomon V, Mitch WE, et al. Muscle protein breakdown and the critical role of the ubiquitin-proteasome pathway in normal and disease states. J Nutr 1999;129:227S-237S.

25. Marzetti E, Carter CS, Wohlgemuth SE, et al. Changes in IL-15 expression and death-receptor apoptotic signaling in rat gastrocnemius muscle with aging and life-long calorie restriction. Mech Ageing Dev 2009; 130:272-280.

26. Meador BM, Pereira SL, Edens NK. Green Tea Supplementation: Current Research, Literature Gaps, and Product Safety. J Food Nutr Disor 2013;2:5.

27. Kerksick CM, Kreider RB, Willoughby DS. Intramuscular adaptations to eccentric exercise and antioxidant supplementation. Amino Acids 2010;39:219-232.

28. Nam S, Smith DM, Dou QP. Ester bond-containing tea polyphenols 


\section{THE JOURNAL OF FRAILTY \& AGING}

potently inhibit proteasome activity in vitro and in vivo. J Biol Chem 2001;276:13322-13330.

29. Quinn LS, Anderson BG, Drivdahl RH, et al. Overexpression of interleukin-15 induces skeletal muscle hypertrophy in vitro: implications for treatment of muscle wasting disorders. Exp Cell Res 2002;280:55-63.

30. Quinn LS, Anderson BG, Strait-Bodey L, et al. Serum and muscle interleukin-15 levels decrease in aging mice: correlation with declines in soluble interleukin-15 receptor alpha expression. Exp Gerontol 2010;45:106-112.

31. Quinn LS, Haugk KL, Grabstein KH. Interleukin-15: a novel anabolic cytokine for skeletal muscle. Endocrinology 1995;136:3669-3672.

32. Sacheck JM, Ohtsuka A, McLary SC, et al. IGF-I stimulates muscle growth by suppressing protein breakdown and expression of atrophyrelated ubiquitin ligases, atrogin-1 and MuRF1. Am J Physiol Endocrinol Metab 2004;287:E591-601.
33. Shen CL, Chyu MC, Yeh JK, et al. Effect of green tea and Tai Chi on bone health in postmenopausal osteopenic women: a 6-month randomized placebo-controlled trial. Osteoporos Int 2012;23:1541-52.

34. Wang H, Lai YJ, Chan YL, et al. Epigallocatechin-3-gallate effectively attenuates skeletal muscle atrophy caused by cancer cachexia. Cancer Lett 2011;305: 40-49.

35. Kung T, Szabo T, Spring J, et al. Cachexia in heart disease: highlights from the ECS 2010. J Cachexia Sarcopenia Muscle 2011; 2:63-69.

36. Koopman R, van Loon LJ. Aging, exercise, and muscle protein metabolism. J Appl Physiol 2009;106:2040-2048.

37. Delp MD, Duan C. Composition and size of type I, IIA, IID/X, and IIB fibers and citrate synthase activity of rat muscle. J Appl Physiol 1996;80:261-270. 